\title{
The building blocks of the universe
}

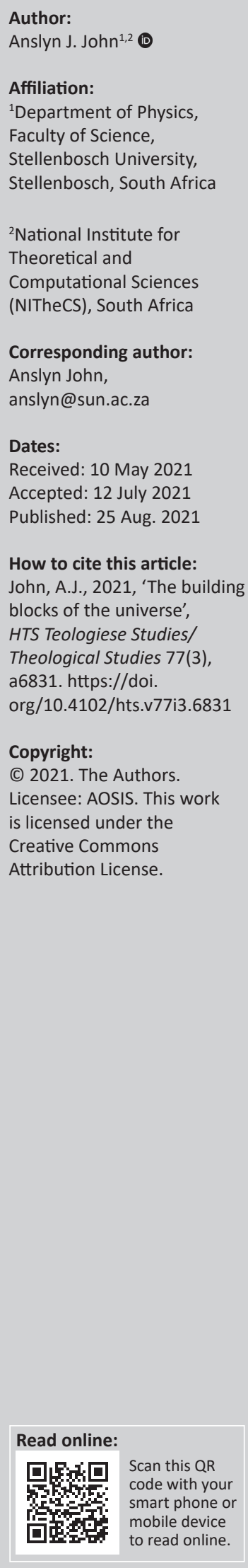

I review the state of knowledge of the composition of the universe for a non-specialist audience. The universe is built up of four components. These are radiation, baryonic (ordinary) matter, dark matter and dark energy. In this article, a quick outline of the theory of Big Bang nucleosynthesis is presented, and the origin of the elements is explained. Cosmology requires the presence of dark matter, which forms most of the mass of the universe, and dark energy, which drives the acceleration of the expansion. The dark sector is motivated, and possible explanations are stated.

Contribution: As part of this special collection on building blocks, the building blocks of the universe are discussed and unsolved problems and proposed solutions are highlighted.

Keywords: cosmology; nucleosynthesis; dark matter; dark energy; cosmological constant; general relativity; galaxies; particle physics.

\section{Introduction}

Cosmology is the scientific study of the origin and development of the universe on the largest scales. Today, the standard model of cosmology is a cornerstone of physics and astronomy. This is attested to by the award of four Nobel Prizes for research in cosmology. These awards, in chronological order, are:

1. Arno A. Penzias and Robert W. Wilson, 1978 - 'for their discovery of cosmic microwave background radiation'.

2. John C. Mather and George F. Smoot, 2006 - 'for their discovery of the blackbody form and anisotropy of the cosmic microwave background radiation'.

3. Saul Perlmutter, Brian P. Schmidt and Adam G. Riess, 2011 - 'for the discovery of the accelerating expansion of the Universe through observations of distant supernovae'.

4. James Peebles, 2019 - 'for theoretical discoveries in physical cosmology'.

Sophisticated models of the universe, making quantitative predictions, were developed soon after the advent of Einstein's theory of general relativity in 1915 (Weinberg 1972). Astronomers had uncovered a universe lacking preferred positions or directions in space. This was in accord with the Copernican principle, which states that we do not occupy a special place in the universe. Relativists naturally incorporated spatial homogeneity and isotropy into their models, but surprisingly discovered that the theory predicted that the universe should be expanding.

An expanding universe was at odds with contemporary observational evidence, and theorists like Einstein attempted to salvage their cosmological models by forcing them to be static. This was abandoned after 1929 when observations of distant galaxies revealed that they were moving away from the Milky Way at speeds proportional to their distances (Kirshner 2004). This empirical formula is now known as the Hubble-Lemaître law (Livio 2011) and follows very naturally from the dynamic cosmological models in general relativity. A picture of a universe that began to expand at some finite time in its past began to emerge. This was later termed the Big Bang model.

The next major development in the history of cosmology was the theory of Big Bang nucleosynthesis (BBN). All of the chemical elements were hypothesised to have been formed by nuclear reactions in the hot, dense infancy of the universe. This was described in the tremendously successful Hot Big Bang model (Gamow 1948). It explained the abundances of many of the chemical elements seen today. It also predicted the existence of relic radiation from the time that the universe became transparent to light. This radiation persisted and was famously observed in the microwave part of the electromagnetic spectrum (Penzias \& Wilson 1965).

Note: Special Collection: Challenging Building Blocks, sub-edited by Chris Jones (Stellenbosch University) and Juri van den Heever (Stellenbosch University). 
Today the standard model of cosmology is known as the $\Lambda \mathrm{CDM}$ or concordance model (Dodelson 2003). It is an extension of the hot Big Bang model, which describes a universe that began to expand 13.8 billion years ago. The early universe was smooth, hot and dense. As it expanded it became cooler and more diffuse and structures such as stars and galaxies started to form. The standard model now includes two extra ingredients viz. dark matter and dark energy. The CDM part represents cold dark matter - a mysterious substance comprising most of the matter of the universe. The $\Lambda$ refers to dark energy, which accounts for most of the universe's energy and is responsible for the acceleration of the universe's expansion at late times. The leading candidate for dark energy is the cosmological constant, which is historically denoted by the Greek letter $\Lambda$.

There are many excellent popular accounts of cosmology, several of which were written by some of the field's leading practitioners (Chown 2010; Hawking 2009; Weinberg 1977). The reader is encouraged to refer to these books for more complete accounts. I will not attempt an exhaustive review of the subject.

Instead, I will focus on addressing the following question in a manner that may be accessible to the enthusiastic layperson: what is the universe made of? The short answer is that the universe is built of four substances: radiation, baryonic matter, dark matter and dark energy.

What are these substances?

1. Radiation: This consists of photons and neutrinos and makes up $10^{-3} \%$ of the total energy.

2. Baryonic matter: This is the ordinary, familiar material including all of the chemical elements and compounds that we find in planets, stars, gas clouds and plasmas. Baryonic matter only comprises about $4 \%$ of the universe's energy budget.

3. Dark matter: This is an exotic substance that does not emit or absorb light. It is not made up of any of the known elementary particles. Dark matter appears to require new physics. It makes up $23 \%$ of the total energy of the universe and comprises most of its mass.

4. Dark energy: This is the name given to the phenomenon responsible for the late time acceleration of the universe's expansion. Like dark matter, this cannot be explained by existing physics and requires extensions to our theories of fundamental particles. Einstein's cosmological constant $(\Lambda)$ is the leading candidate for dark energy, which makes up $73 \%$ of today's energy budget.

Dividing the building blocks of the universe into these four substances is convenient as each of these substances contributes to the total energy of the universe. Moreover, each of these substances affects the overall dynamics of the universe in different ways at particular times. The early universe is dominated by radiation (photons and neutrinos). Photons lack mass but possess energy. During the universe's infancy, most of its energy resides in its photons. This is the so-called radiation era. As the universe expands the energy density of its constituents decreases because the same particles occupy an increasingly larger volume. The radiation energy density declines at a faster rate than the matter energy density. After a period termed matter-radiation equality, the dominant contribution to the energy density of the universe comes from the matter sector. The matter sector comprises dark matter and baryonic matter. This is the start of the matter era. Although the energy density of the matter particles continues to drop as the universe expands, it continues to dominate the energy density of the radiation sector, which drops at an even faster rate. Eventually the energy density of the matter particles drops below the energy density of the dark energy component. After this time, the universe is dominated by dark energy and starts to expand exponentially. This is in contrast to the earlier radiation and matter eras where the universe experienced power law expansion.

In the next section I will describe how the ordinary matter was formed and how radiation from this process persisted. I will then describe dark matter and dark energy before concluding.

\section{Big Bang nucleosynthesis}

The ordinary matter we are familiar with is composed of chemical elements. These range in mass from the lightest elements, hydrogen and helium, to the heaviest naturally occurring elements like uranium. The basic properties of these elements are described by the periodic table, ascribed to Mendeleev. For more details about the building blocks of matter, the reader is referred to the relevant article (Haynes \& Blackie 2021) in this edition of the journal.

The science of how the atoms of these elements combine to form molecules and compounds in nature and the laboratory is the purview of chemistry. Explaining the origin of these elements is the task of physics. Although individual atoms may gain or lose electrons, the fundamental characteristics of an element are determined by the number of protons in their nuclei.

Nuclei are composed of positively charged protons and electrically neutral neutrons. A neutron is slightly heavier than a proton, and both of these particles are about 2000 times heavier than an electron. An electron has a negative charge, which perfectly balances the positive charge of a proton. As a neutral atom has the same number of electrons and protons, the nucleus essentially contains all of the mass of an atom.

In nuclear and particle physics the equivalence of mass and energy is a vital concept. This is a consequence of Einstein's famous formula, $\mathrm{E}=m c^{2}$, where $c=3 \times 10^{8} \mathrm{~m} / \mathrm{s}$ is the speed of light, a fundamental constant of nature. It is convenient to measure the masses of subatomic particles in energy units. In this system of units, the proton mass is 938.3 million $\mathrm{eV}$ whilst the neutron weighs 939.6 million $\mathrm{eV}$. The electron-Volt $(\mathrm{eV})$ is 
a commonly used unit of energy and one needs a fairly small amount of energy viz. $13.6 \mathrm{eV}$ to ionise a hydrogen atom by 'knocking off' a single electron. Because the binding energy of a typical nucleus is vastly larger than the ionisation energy of typical atom, it is significantly more difficult to break up a nucleus than it is to break up an atom. This difference in binding energies accounts for the enormous difference in scale between 'electrical' energy and nuclear energy.

To build heavier elements from lighter elements one needs to combine nuclei. Once formed, these nuclei can bind with electrons from their surroundings to form neutral atoms. Successive elements in the periodic table are characterised by increasing numbers of protons. One cannot simply combine two protons because they are positively charged particles and like charges repel. The electric repulsion between like charges can however be overcome by nuclear forces. Stable nuclei thus require neutrons as well as protons. Working out exactly how this process, nucleosynthesis, happens is the nontrivial task of the nuclear physicist.

The quest to explain the origin of the chemical elements lasted several decades and forms one of the greatest triumphs of 20th-century science. There were many bold leaps and false starts. I will not recount the fascinating history of this journey but will describe the key discoveries. By the end of the 1960s a consistent picture had emerged: lighter elements such as hydrogen, helium and lithium were formed during the Big Bang whilst heavier elements such as carbon, nickel and iron were formed much later in stars.

What became known as the Hot Big Bang model of cosmology originated with a series of seminal articles by George Gamow, Ralph Alpher and Robert Hermann (Alpher, Bethe \& Gamow 1948; Alpher \& Herman 1948, 1949, 1950; Gamow 1948). According to the Friedmann-Lemaittre-Robertson-Walker models in general relativity, the average temperature of the universe decreases as it expands. Further back in the past, the universe would have been hotter and more energetic. At temperatures above $10^{10} \mathrm{~K}$ the photons are so energetic that nuclear reactions cannot proceed. Nuclear reactions producing free protons and nuclei only begin once the temperature drops to $10^{9} \mathrm{~K}$.

Whilst protons are stable particles, neutrons have a half-life of about $15 \mathrm{~min}$. Once formed, neutrons will rapidly decay into protons, electrons and anti-neutrinos. Before this process, beta decay, happens the neutrons can interact with protons to form deuterons. A deuteron is a nucleus containing one proton and one neutron. When a deuteron binds to an electron, it forms deuterium, a known isotope of hydrogen. Gamow calculated that a fraction of free neutrons will merge with free protons to form deuteron nuclei, which are stable. Once these deuteron nuclei have formed they can interact with free protons in a succession of nuclear reactions to produce the nuclei of helium, lithium and other light elements. All of the neutrons that we see in every atom today had to have been smuggled in from the early universe in deuteron nuclei.
All of these nuclear reactions occurred in the early universe, which proceeded to expand and cool. Eventually the temperature dropped below $3000 \mathrm{~K}$ and the photons became sufficiently cool that they could no longer ionise hydrogen. The hydrogen nuclei could then attract electrons to form hydrogen atoms. This event is known as recombination. Once this happened the photons stopped interacting with matter and the universe became transparent to light for the first time. The radiation that was emitted at this time survived to the present day and is observed as the cosmic microwave background radiation. Gamow and his collaborators predicted that this radiation survived and would glow at a temperature of about $10 \mathrm{~K}$ today.

Apart from the early universe, nucleosynthesis naturally occurs in stars. Hans Bethe explained the energy output of stars as a result of a series of nuclear reactions where hydrogen is 'burned' to helium (Bethe 1939). The mechanisms to generate most of the heavier elements in stars were outlined by Margaret Burbidge, Geoffrey Burbidge, William A. Fowler and Fred Hoyle (Burbidge et al. 1957). This seminal article is often referred to $\mathrm{B}^{2} \mathrm{FH}$ after the initials of its authors. Some of these mechanisms describe the creation of the heaviest elements in stellar explosions such as supernovae. These events also deposit the heaviest elements in the interstellar medium where they can be recycled into younger stars and planetary systems.

Hoyle was a proponent of the soon-to-be falsified Steady State model of cosmology. The popular name of the rival cosmology, the Big Bang model, was derisively coined by Hoyle. The $\mathrm{B}^{2} \mathrm{FH}$ stellar nucleosynthesis scenario could not explain the observed abundances of helium in stars. Using an energy argument, Hoyle concluded that the helium observed in today's stars could not have been produced by stellar nucleosynthesis and must have been present before the first stars had formed. Attention then returned to BBN, which could naturally explain the observed helium abundances.

The story of nucleosynthesis does not end in 1957. The debate between which of the several processes outlined in $\mathrm{B}^{2} \mathrm{FH}$ was responsible for the creation of the heavy elements such as uranium, platinum and gold was only settled in 2017. In that year, the LIGO and VIRGO observatories detected gravitational wave signals from the merger of two neutron stars (Abbott et al. 2017). From studies of the electromagnetic waves that were also emitted during this event, scientists determined that these heavy elements were produced by the merger of neutron stars.

Recall that BBN also predicted the existence of relic radiation from the recombination period. The photons emitted at that period would have created a spectrum of blackbody radiation. In 1965, this relic radiation was serendipitously detected at a temperature of $2.7 \mathrm{~K}$ by Arno Penzias and Robert Wilson whilst they were observing microwave emissions from the galaxy. Remarkably the Princeton team of Robert Dicke, James Peebles, David Wilkinson and Peter Roll was aiming to discover the cosmic microwave background 
radiation when the nearby team of Penzias and Wilson at Bell Laboratories revealed its findings. The two groups published their results in the same issue of the Astrophysical Journal (Dicke et al. 1965; Penzias \& Wilson 1965), and a new era in observational cosmology was born.

In 1992 the Cosmic Orbital Background Explorer (COBE) team announced the discovery of ripples in the spectrum of the microwave background radiation (Smoot et al. 1992). These ripples represent small fluctuations in the temperature of the relic photons. These temperature fluctuations were caused by slight variations in the density of matter at the time of recombination. These density perturbations would later grow and form the sites where larger structures such as galaxies and clusters could form. The cosmic microwave background is the oldest fossil ever found and functions as a scientific Rosetta stone. It continues to translate the physics of our distant past into the astronomy of our present (Hinshaw et al. 2013).

\section{Dark matter}

\section{Astronomical evidence}

Strong evidence for the existence of dark matter comes from studies of the dynamics of the Milky Way galaxy.

Before outlining these arguments, I present some basic facts about our galaxy. The Milky Way is a typical spiral galaxy with the appearance of a flattened, rotating disk (Binney \& Tremaine 2011). In astronomers' units of measurement, its mass is approximately $10^{12} M_{\odot}$ meaning that the galaxy weighs as much as 1000000000000 Suns. The Milky Way has a diameter of about 185000 light years and hosts between 100 and 400 billion stars as well as large amounts of gas. The Sun is a typical star and resides in one of the spiral arms at a distance of 27000 light years from the galactic centre. The Sun orbits the galactic centre once every 240 million years. The centre of the galaxy hosts a supermassive black hole weighing $4 \times 10^{6} M_{\odot}$. Remarkably, the stars, black holes and other distributions of gas and rock make up only a tiny fraction of the mass of our galaxy. Most of the mass is made up of dark matter. This will turn out to have important implications for cosmology as well as galactic astronomy.

How do we know that most of the galaxy's mass comes from dark matter? The argument follows from Kepler's laws of planetary motion, which were originally used to describe the orbits of planets in the solar system (Vogt 1996; Young et al. 1996). These laws can ultimately be derived from Newton's inverse square law of gravity. By virtue of the universal character of gravity, Kepler's laws apply just as easily to the motion of planets around stars as they do to the motion of stars around the centres of galaxies.

According to Kepler's third law, the period of revolution, $T$, of a body orbiting another body of mass, $M$, is related to the distance, $r$, between these bodies by the formula:
$T^{2}=\left(\frac{4 \pi^{2}}{G M}\right) r^{3}$

[Eqn 1]

In this equation, $\mathrm{G}=6.67 \times 10^{-11} \mathrm{~m}^{3} \mathrm{Kg}^{-1} \mathrm{~s}^{-2}$ and represents Newton's universal gravitational constant. During a single revolution about the Galactic centre, a star covers a distance given by the circumference, $2 \pi \mathrm{r}$, of its orbit. Its speed, $v$, is simply the distance covered divided by the time taken:

$v=2 \pi r / T$.

[Eqn 2]

Combining the last two equations we obtain the following relation between the star's speed and its distance from the centre of the galaxy:

$v=\sqrt{\frac{G M}{r}}$.

[Eqn 3]

The tangential velocity, $v$, of a star is inversely proportional to the square root of its distance from the object it orbits, that is,

$v \alpha r^{-1 / 2}$

[Eqn 4]

The further the star is from the centre of the galaxy, the smaller its velocity.

Quite unexpectedly, this is not what happens in our Milky Way! Whilst the velocity-distance relation holds in our solar system, it fails on galactic scales. Astronomers have observed that stellar velocities remain fairly constant as the distance from the centre increases - the rotation curves are substantially more flat than predicted. Have Kepler's laws failed at the scale of the galaxy despite working so well at the scale of the solar system?

Flat rotation curves have been observed in other galaxies (Rubin \& Ford 1970). This behaviour is not unique to our own galaxy. How do we explain the ubiquity of flat rotation curves? The Keplerian analysis would still be valid if, instead of having the bulk of the galaxy's mass concentrated at the centre, the mass distribution increased linearly as a function of radius.

So, where is all of this extra mass? We would need quite a lot of it in order for galaxies to look the way they do. If we add up all of the mass from the stars and gas that we observe in our galaxy, that total only accounts for $10 \%$ of what we require to produce the observed rotation curves. Most of the mass of a galaxy is non-luminous or dark, and this mass makes its influence felt by its gravitational field. The Milky Way should be thought of as a flattened disk of visible stars and gas surrounded by a spherical halo of invisible, dark matter.

Galaxies are not isolated objects but belong to clusters, which are large, gravitationally bound systems of galaxies. A typical cluster has about a 100 galaxies and a radius of roughly $3 \times 10^{6}$ light years. Individual galaxies revolve about the cluster's centre of mass and one can measure rotation curves of clusters 
in a manner analogous to measurements of stellar rotation curves within individual galaxies. As with galaxies, clusters exhibit a flattening of their rotation curves. This can be explained if most of the mass in clusters comes from dark matter. Fritz Zwicky invoked what he termed 'dunkle (kalt) Materie' or dark matter to explain the excessive velocities of the galaxies he observed in the Coma Cluster (Zwicky 1933, 1937). This is the first known historical reference to dark matter.

It is important to note that galactic rotation curves are predicted by assuming the validity of Newton's theory of gravity. Could one obviate the need for dark matter by explaining the observed behaviour by using Einstein's theory of gravity (general relativity) instead? Whilst general relativity is a successful and epistemologically wealthier theory of gravity, it necessarily encompasses Newtonian gravity (Carroll 2019; Weinberg 1972). In the limit of weak gravitational fields and slow-moving bodies the predictions of general relativity are indistinguishable from those of Newtonian gravity. At the scale of galaxies, the use of Newtonian theory is perfectly justifiable, and the presence of dark matter is unavoidable.

Even when one examines systems with gravitational fields that have to be modelled by using general relativity, the presence of dark matter is still required. In general relativity, matter and energy dynamically interact with spacetime. What we perceive as the gravitational force is a consequence of matter and energy distorting spacetime. This was succinctly described by John Wheeler when he stated, 'Spacetime tells matter how to move, matter tells spacetime how to curve' (Misner et al. 1973).

Rays of light travel along particular paths in spacetime. When these rays pass close to regions of spacetime that have been distorted (e.g., by a dense concentration of matter or energy), they change their trajectory. As a result, an observer will detect an image of a star or galaxy that has been displaced from its true position in the sky. This phenomenon is known as gravitational lensing (Wambsganss 1998). In optics, the appearance of mirages and bent shapes of pencils in glasses of water can be accurately predicted (Young et al. 1996). Similarly, the changes in positions of stars and galaxies can be predicted by general relativity. This novel effect was predicted by Einstein and forms one of the first tests of the general theory of relativity. The bending of light rays from distant stars by the Sun was famously observed by Eddington and his team during the solar eclipse of 1919 (Eddington 1919).

Images of gravitationally lensed galaxies have also been observed (Walsh, Carswell \& Weymann 1979). Multiple images of the same galaxy can be seen when light rays are deflected by a cluster sitting directly between the galaxy and an observer. From the general relativistic formula, one can deduce the mass of the lense, that is the cluster. This mass value is much lower than that inferred from the visible light from the cluster. Most of the mass in clusters has to be in the form of dark matter.
The case for dark matter is further enhanced when one analyses the spectrum of the cosmic microwave background radiation (Hinshaw et al. 2013), the relic radiation from the time that the universe became transparent to light. All of these pieces of evidence consistently point towards a universe where most of the matter is dark.

\section{Dark matter candidates}

It has been established that large quantities of dark matter exist and interact gravitationally but not electromagnetically (because dark matter does not emit light). What remains to be determined is the identity of dark matter. The two main classes of dark matter candidates are known as weakly interacting massive particles (WIMPs) and massive compact halo objects (MACHOs) (Bertone 2010).

Weakly interacting massive particles are subject to the weak force, which is one of the four fundamental forces in nature, the other three being gravity, electromagnetism and the strong nuclear force. Neutrinos are firmly established elementary particles that interact via the weak force and gravity, and it will be instructive to examine their properties as a proxy for WIMPs.

Neutrinos were hypothesised by Wolfgang Pauli in 1930 to resolve the apparent violations of energy, momentum and angular momentum conservation observed in beta decay (Brown 1978). They were long believed to have zero rest mass (like their well-known massless relatives, the photons). Neutrinos were finally detected in 1956 (Cowan et al. 1956). The determination of their physical properties, including their rest masses, is still incomplete and remains an active area of theoretical and empirical research (Dolgov 2002).

Neutrinos interact with other particles either gravitationally or through the electroweak force. The electroweak force (which arises from the unification of electromagnetism and the weak force) has a small interaction cross-section, which makes neutrinos very difficult to detect. Enormous amounts of neutrinos were created by nuclear reactions in the hot, dense early universe. Despite their abundance, neutrinos have a very small probability of interacting with other forms of matter. Billions of neutrinos pass through the Earth every second and barely an impression is made.

There are believed to be only three flavours of neutrino, viz. the electron neutrino $\left(v_{e}\right)$, the muon neutrino $\left(\mathrm{v}_{\mu}\right)$ and the tau neutrino $\left(\mathrm{v}_{\tau}\right)$ as well as their respective anti-particles $\left(\bar{v}_{e}, \bar{v}_{\mu}\right.$ and $\left.\bar{v}_{\tau}\right)$. All three flavours have been detected. The known neutrino species cannot, by themselves, account for the dark matter in the universe.

According to particle physics models, WIMPs could be produced in the universe's infancy when the temperature was very high. Weakly interacting massive particles are a class of hypothetical weakly interacting particles that could comprise all of the dark matter if their rest masses fell in the range between $1 \mathrm{GeV}$ and $1 \mathrm{TeV}$, that is between $10^{9}$ and $10^{12} \mathrm{eV}$. 
According to this scenario WIMPs are initially in thermal equilibrium with the photons and would have roughly the same number densities, that is the same number of particles per unit volume.

As the universe expands, its average temperature $(T)$ decreases and the particle number densities drop. The WIMP and photon number densities will decrease in the same ratio. When the universe's temperature drops below the value ( $E \sim k T$, where $\mathrm{K}$ is Boltzmann's fundamental constant) corresponding to the mass energy of the WIMP $\left(E \sim m c^{2}\right)$, the creation of new WIMPs is suppressed. The remaining particles can still annihilate and the overall effect of these two processes is that the WIMP number density drops exponentially, and the WIMPs are no longer in thermal equilibrium with the photons. Eventually, the density becomes sufficiently low that the probability of a WIMP encountering and annihilating another particle becomes very small. The surviving particles, which are no longer being created or annihilated, now constitute what we call dark matter (Kolb \& Turner 1994).

From the observed density of dark matter in the universe today, one can infer the interaction strength of the WIMPs that may have produced these particles. The WIMP interaction strength turns out to correspond to the electroweak scale. This scale is within reach of particle accelerators such as the Large Hadron Collider (LHC) and proton colliders at Fermilab. These experiments could therefore create previously undiscovered WIMPs, which would be the same type of particle needed to explain all of the dark matter in the universe.

Theoretical high energy physicists have proposed an extension to the standard model of particle physics based on supersymmetry. If supersymmetry were true, every subatomic particle would have a supersymmetric partner (Fayet \& Ferrara 1977). Examples of these partners include neutralinos, photinos, Higgsinos, sleptons and squarks. The discovery of a supersymmetric particle will potentially identify new WIMPs as the dark matter particle. To date no experimental evidence for supersymmetry has emerged (Wolchover 2012).

Massive compact halo objects form the other broad class of dark matter candidates. Galactic halos could be filled with Jupiter-mass objects weighing up to one-thousandth the mass of the Sun $\left(M \sim 10^{-3} M_{\odot}\right)$ or brown dwarf stars $\left(M \sim 8 \times 10^{-2} M_{\odot}\right)$. Primordial black holes that may have been formed in the early universe are another potential MACHO. All of these objects evade direct detection as they do not emit electromagnetic radiation. Massive compact halo objects can however be detected via microlensing (Roulet \& Mollerach 1997), which is based on the principle of gravitational lensing. Intense observational efforts in this direction are underway (Niikura et al. 2019).

Weakly interacting massive particles and MACHOs are amongst the more orthodox explanations for dark matter. Scientists have examined the possibility that the gravitational force could deviate from the inverse square law on galactic scales. This is the basis of the modified Newtonian dynamics (MOND) paradigm (Sanders \& McGaugh 2002). Modifications of the relativistic gravitational theory have also been proposed (Bekenstein 2004; Moffat 2006). A common shortcoming of many modified theories of gravity is the fact that they struggle to consistently explain all of the relevant astronomical observations. Successfully replacing dark matter with an alternative theory of gravity remains a formidable task (Pardo \& Spergel 2020).

\section{Dark energy}

This is the largest and, by far, least well-understood component. In the 1990s two rival teams of astronomers measured the intensity and redshifts of supernovae. Supernovae form when certain types of stars explode. For a short period they emit vast amounts of radiation: a single supernova can appear as bright as its host galaxy.

Distance measurements are a persistent problem in astronomy. Fortunately, studies of type Ia supernovae have established that they form a specific class of supernovae whose uniform behaviour permits an accurate determination of their intrinsic brightness. An object in the sky has an apparent brightness because of a combination of its intrinsic brightness and its distance from the observer. The uniformity of type Ia supernovae makes them excellent standard candles. Because we know how intrinsically bright they are, we can determine how far away they are from measurements of their apparent brightness. Supernovae also have the advantage of being extremely luminous making them much easier to see at great distances than other potential standard candles.

Supernova observations are, however, quite challenging. They are quite rare: on average a galaxy experiences only one supernova event every 1000 years. They are also hard to predict: we have little indication of when a star will go supernova, and we do not know in advance where or when this will happen. In addition, they are short-lived. Once a supernova occurs, its intensity rapidly drops over a period of weeks and it becomes too faint to study.

Despite these considerable challenges the High-Z Supernova Search Team and the Supernova Cosmology Project discovered a number of type Ia supernovae and determined their brightness and distances (Perlmutter et al. 1999; Riess et al. 1998). The distance measurements can be made by analysing the redshift of the spectra of each supernova. This is based on the Doppler effect, a well-known example of which is the compression of sound waves arriving from a train moving towards us, and the stretching of these sound waves as the train moves away from us. Changes in the wavelength of sound waves lead to discernible changes in the pitch of the sound, and we can calculate how far away the train is. A similar sort of analysis can be conducted for light from a distant star or galaxy. Most distant galaxies are moving away from us, and the wavelengths of the light waves emitted by those galaxies will become stretched or 'redshifted'. 
In 1998 the two supernovae studies uncovered a surprising result. Analysis of the data revealed that distant galaxies were not simply receding from us at speeds proportional to their distance from us (the Hubble-Lemaitre law), but that they were also starting to accelerate. This acceleration was observed to occur at relatively recent times. This is in stark contrast to the more regular expansion of the universe over most of its history.

The cosmic acceleration cannot be explained by a universe containing only radiation and matter (either ordinary or dark). Whatever was responsible for this late time acceleration makes up $70 \%$ of the universe's energy today. Michael Turner is credited with coining the term dark energy for this mysterious new arrival in the cosmic inventory (Huterer \& Turner 1999).

The supernova observations were surprising but consistent with, and subsequently corroborated by, other analyses based on the cosmic microwave background radiation and surveys of large-scale structures. The leaders of the original supernova studies (Saul Perlmutter, Brian Schmidt and Adam Riess) were awarded the 2011 Nobel Prize in Physics. Dark energy, whatever it may be, is here to stay.

What is responsible for the acceleration? The simplest explanation as well as the one currently favoured by statistical analysis is the cosmological constant, $\Lambda$. This is believed to be an energy component intrinsic to the vacuum of spacetime, which has been predicted by quantum field theory. The discord between the theoretical value of the vacuum energy and the observed value of $\Lambda$ inferred from astronomical observations is however enormous (Weinberg 1989). This is one of the main reasons why the cosmological constant has not been universally accepted as the source of dark energy.

As with dark matter, one has to invoke exotic extensions to the standard model of particle physics to explain dark energy. Some of the possible candidates include a dynamical, timevarying cosmological constant, scalar fields or radical modifications of Einstein's theory of general relativity. At the time of writing, observational data are not sufficiently precise to conclusively discriminate between these competing hypotheses. Many observational programmes are underway in the hope of solving this mystery.

\section{Conclusion}

The building blocks of the universe are four classes of substance: radiation, baryonic matter, dark matter and dark energy. Radiation and ordinary matter are well understood but make up only $4 \%$ of the energy of the universe. The dark sector, comprising dark matter and dark energy, constitutes $96 \%$ of the energy of the universe today. New advances in physics are required to explain the dark sector. The modern picture of the universe is consistently derived from the successful application of many areas of physics and astronomy across a wide range of energy, time and distance scales. The subject is as dynamic as the universe itself, and new discoveries and mysteries lie in wait.

\section{Acknowledgements Competing interests}

The author declares that he has no financial or personal relationships that may have inappropriately influenced him in writing this article.

\section{Author's contributions}

A.J.J. is the sole author of this article.

\section{Ethical considerations}

This article followed all ethical standards for research without direct contact with human or animal subjects.

\section{Funding information}

This research was funded by Stellenbosch University.

\section{Data availability}

Data sharing is not applicable to this article as no new data were created or analysed in this study.

\section{Disclaimer}

The views and opinions expressed in this article are those of the author and do not necessarily reflect the official policy or position of any affiliated agency of the author.

\section{References}

Abbott, B.P., Abbott, R., Abbott, T.D., Acernese, F., Ackley, K., Adams, C. et al., 2017, 'GW170817: Observation of gravitational waves from a binary neutron star inspiral', Physical Review Letters 119(16), 161101. https://doi.org/10.1103/ PhysRevLett.119.161101

Alpher, R.A., Bethe, H. \& Gamow, G., 1948, 'The origin of chemical elements', Physical Review 73, 803. https://doi.org/10.1103/PhysRev.73.803

Alpher, R.A. \& Herman, R., 1948, 'Evolution of the universe', Nature 162, 774. https:// doi.org/10.1038/162774b0

Alpher, R.A. \& Herman, R.C., 1950, 'Theory of the origin and relative abundance distribution of the elements', Reviews of Modern Physics 22, 153. https://doi. org/10.1103/RevModPhys.22.153

Alpher, R.A. \& Herman, R.C., 1949, 'Remarks on the evolution of the expanding universe', Physical Review 75, 1089. https://doi.org/10.1103/PhysRev.75.1089

Bekenstein, J.D., 2004, 'Relativistic gravitation theory for the modified Newtonian dynamics paradigm', Physical Review D 70, 083509. https://doi.org/10.1103/ PhysRevD.70.083509

Bertone, G., 2010, 'The moment of truth for WIMP dark matter', Nature 468, 389. https://doi.org/10.1038/nature09509

Bethe, H.A., 1939, 'Energy production in stars', Physical Review 55, 434. https://doi. org/10.1103/PhysRev.55.434

Binney, J. \& Tremaine, S., 2011, Galactic dynamics, Princeton University Press, Princeton, NJ.

Brown, L.M., 1978, 'The idea of the neutrino', Physics Today 31(9), 23. https://doi. org/10.1063/1.2995181

Burbidge, E.M., Burbidge, G.R., Fowler, W.A. \& Hoyle, F., 1957, 'Synthesis of the elements in stars', Reviews of Modern Physics 29, 547. https://doi.org/10.1103/ RevModPhys.29.547

Carroll, S.M., 2019, Spacetime and geometry, Cambridge University Press, Cambridge.

Chown, M., 2010, Afterglow of creation: Decoding the message from the beginning of time, Faber \& Faber, London.

Cowan, C.L., Reines, F., Harrison, F., Kruse, H. \& McGuire, A., 1956, 'Detection of the free neutrino: A confirmation', Science 124(3212), 103. https://doi.org/10.1126/ science.124.3212.103

Dicke, R.H., Peebles, P.J.E., Roll, P.G. \& Wilkinson, D.T., 1965, 'Cosmic black-body radiation', The Astrophysical Journal 142, 414. https://doi.org/10.1086/148306

Dodelson, S., 2003, Modern cosmology, Elsevier, Amsterdam. 
Dolgov, A.D., 2002, 'Neutrinos in cosmology', Physics Reports 370, 333. https://doi. org/10.1016/S0370-1573(02)00139-4

Eddington, A.S., 1919, 'The total eclipse of 1919 May 29 and the influence of gravitation on light', The Observatory 42, 119.

Fayet, P. \& Ferrara, S., 1977, 'Supersymmetry', Physics Reports 32(5), 249. https://doi. org/10.1016/0370-1573(77)90066-7

Gamow, G., 1948, 'The evolution of the universe', Nature 162, 680. https://doi. org/10.1038/162680a0

Hawking, S., 2009, A brief history of time: From big bang to black holes, Random House, New York, NY.

Haynes, D.A. \& Blackie, M.A.L., 2021, 'The building blocks of matter', HTS Teologiese Studies/Theological Studies 77(3), a6809. https://doi.org/10.4102/hts.v77i3.6809

Hinshaw, G., Larson, D., Komatsu, E., Spergel, D.N., Bennett, CL., Dunkley, J. et al. 2013, 'Nine-year Wilkinson Microwave Anisotropy Probe (WMAP) observations: Cosmological parameter results', The Astrophysical Journal Supplement Series 208(2), 19. https://doi.org/10.1088/0067-0049/208/2/19

Huterer, D. \& Turner, M.S., 1999, 'Prospects for probing the dark energy via supernova distance measurements', Physical Review D 60(8), 081301. https://doi. org/10.1103/PhysRevD.60.081301

Kirshner, R.P., 2004, 'Hubble's diagram and cosmic expansion', Proceedings of the National Academy of Sciences 101(1), 8-13. https://doi.org/10.1073/ pnas.2536799100

Kolb, E. \& Turner, M., 1994, The early universe, CRC Press, Boca Raton.

Livio, M., 2011, 'Mystery of the missing text solved', Nature 479, 171. https://doi. org $/ 10.1038 / 479171$ a

Misner, C.W., Thorne, K.S. \& Wheeler, J.A., 1973, Gravitation, W.H. Freeman and Company, New York.

Moffat, J.W., 2006, 'Scalar-tensor-vector gravity theory', Journal of Cosmology and Astroparticle Physics 2006, 004. https://doi.org/10.1088/1475-7516/2006/03/004

Niikura, H., Takada, M., Yokoyama, S., Sumi, T. \& Masaki, S., 2019, 'Constraints on Earth-mass primordial black holes from OGLE 5-year microlensing events', Physical Review D 99(8), 083503. https://doi.org/10.1103/PhysRevD.99.083503

Pardo, K. \& Spergel, D.N., 2020, 'What is the price of abandoning dark matter? Cosmological constraints on alternative gravity theories', Physical Review Letters 125(21), 211101. https://doi.org/10.1103/PhysRevLett.125.211101

Penzias, A.A. \& Wilson, R.W., 1965, 'A measurement of excess antenna temperature at $4080 \mathrm{Mc} / \mathrm{s}^{\prime}$, The Astrophysical Journal 142, 419 . https://doi. org/10.1086/148307
Perlmutter, S., Aldering, G., Goldhaber, G., Knop, R., Nugent, P., Castro, P.G. et al., 1999, 'Measurements of $\Omega$ and $\wedge$ from 42 high-redshift supernovae', The Astrophysical Journal 517(2), 565. https://doi.org/10.1086/307221

Riess, A.G., Filippenko, A.V., Challis, P., Clocchiatti, A., Diercks, A., Garnavich, P.M. et al., 1998, 'Observational evidence from supernovae for an accelerating universe and a cosmological constant', The Astronomical Journal 116(3), 1009. https://doi. org/10.1086/300499

Roulet, E. \& Mollerach, S., 1997, 'Microlensing', Physics Reports 279(2), 67. https:// doi.org/10.1016/S0370-1573(96)00020-8

Rubin, V.C. \& Ford, Jr., W.K., 1970, 'Rotation of the Andromeda nebula from a spectroscopic survey of emission regions', The Astrophysical Journal 159, 379. https://doi.org/10.1086/150317

Sanders, R.H. \& McGaugh, S.S., 2002, 'Modified Newtonian dynamics as an alternative to dark matter', Annual Review of Astronomy and Astrophysics 40, 263. https:// to dark matter', Annual Review of Astronomy and
doi.org/10.1146/annurev.astro.40.060401.093923

Smoot, G.F., Bennett, C.L., Kogut, A., Wright, E., Aymon, J., Boggess, N. et al., 1992, 'Structure in the COBE differential microwave radiometer first-year maps', The Astrophysical Journal 396, L1. https://doi.org/10.1086/186504

Vogt, E., 1996, 'Elementary derivation of Kepler's laws', American Journal of Physics 64(4), 392. https://doi.org/10.1119/1.18253

Walsh, D., Carswell, R.F. \& Weymann, R.J., 1979, '0957+ 561 A, B: Twin quasistellar objects or gravitational lens?', Nature 279, 381. https://doi.org/10.1038/279381a0

Wambsganss, J., 1998, 'Gravitational lensing in astronomy', Living Reviews in Relativity 1, 1. https://doi.org/10.12942/Irr-1998-12

Weinberg, S., 1972, Gravitation and cosmology: Principles and applications of the general theory of relativity, Wiley, New York, NY.

Weinberg, S., 1977, The first three minutes. A modern view of the origin of the universe, Basic Books, viewed n.d, from https://www.basicbooks.com/.

Weinberg, S., 1989, 'The cosmological constant problem', Reviews of Modern Physics 61(1). https://doi.org/10.1103/RevModPhys.61.1

Wolchover, N., 2012, 'As Supersymmetry Fails Tests, Physicists seek new Ideas', Quanta Magazine, November 20, viewed n.d., from http://www. simonsfoundation.org/quanta/20121120-as-supersymmetry-fails-testsphysicists-seek-new-ideas/.

Young, H.D., Freedman, R.A., Sandin, T. \& Ford, A.L., 1996, University physics, AddisonWesley, Reading, MA.

Zwicky, F., 1933, 'The redshift of extragalactic nebulae', Helvetica Physica Acta 6, 110.

Zwicky, F., 1937, 'On the masses of nebulae and of clusters of nebulae', The Astrophysical Journal 86, 217. https://doi.org/10.1086/143864 